\title{
Enhancement of long-range correlations in a 2D vortex lattice by an incommensurate 1D disorder potential
}

\author{
I. Guillamón ${ }^{1,2,3 \star}$, R. Córdoba ${ }^{4,5 \dagger}$, J. Sesé ${ }^{4,5}$, J. M. De Teresa ${ }^{4,5,6}$, M. R. Ibarra ${ }^{4,5}$, S. Vieira ${ }^{1,2}$ \\ and $H$. Suderow ${ }^{1,2}$
}

Long-range correlations in two-dimensional (2D) systems are significantly altered by disorder potentials. Theory has predicted the existence of disorder-induced phenomena, such as Anderson localization ${ }^{1}$ or the emergence of a Bose glass ${ }^{2}$. More recently, it has been shown that when disorder breaks 2D continuous symmetry, long-range correlations can be enhanced ${ }^{3}$. Experimentally, developments in quantum gases have allowed the observation of the effects of competition between interaction and disorder ${ }^{4,5}$. However, experiments exploring the effect of symmetry-breaking disorder are lacking. Here, we create a 2D vortex lattice at $0.1 \mathrm{~K}$ in a superconducting thin film with a well-defined 1D thickness modulation-the symmetry-breaking disorder-and track the field-induced modification using scanning tunnelling microscopy. We find that the 1D modulation becomes incommensurate with the vortex lattice and drives an order-disorder transition, behaving as a scale-invariant disorder potential. We show that the transition occurs in two steps and is mediated by the proliferation of topological defects. The resulting critical exponents determining the loss of positional and orientational order are far above theoretical expectations for scale-invariant disorder ${ }^{6-8}$ and follow instead the critical behaviour describing dislocation unbinding melting ${ }^{9,10}$. Our data show that randomness disorders a 2D crystal, with enhanced long-range correlations due to the presence of a 1D modulation.

The competition between order and disorder is a fundamental problem in condensed-matter physics, which directly impacts many different systems, such as crystalline solids ${ }^{7,11}$, electronic or magnetic arrangements ${ }^{12}$, localization in metals and superconductors, or vortex lattices in superconductors and condensates ${ }^{4,13}$. In 2D systems, long wavelength fluctuations induce deviations in the atomic positions from the perfect lattice, with the mean-squared displacement diverging logarithmically at large distances ${ }^{14}$. One major consequence is the so-called MerminWagner-Hohenberg $(\mathrm{MWH})$ theorem ${ }^{14,15}$, which states that no true order exists in 2D systems at any finite temperature. Usually, we can distinguish between static quenched disorder and fluctuations. In the absence of quenched disorder, thermal fluctuations drive the $2 \mathrm{D}$ melting transition which is described by Berezinskii-Kosterlitz-
Thouless-Halperin-Nelson-Young (BKTHNY) theory through the two-stage proliferation and unbinding of topological defects ${ }^{9,10,16,17}$. Quenched disorder, on the other hand, is expected to suppress long-range correlations more effectively than temperature ${ }^{18}$. It can be classified as pinning with identifiable length scales, such as impurities or defects in 2D crystals, or as scale-invariant (random) disorder, as for example in an amorphous film. Pinning destroys long-range $2 \mathrm{D}$ correlations at any strength ${ }^{19,20}$. Scale-invariant disorder produces power-law decaying correlations and a transition to a disordered lattice with exponentially falling correlations above a critical disorder strength ${ }^{6,7}$. The order-disorder transition induced by scale-invariant disorder has been investigated in a wide range of physical systems, such as $2 \mathrm{D}$ disordered XY models ${ }^{6}, 2 \mathrm{D}$ solids ${ }^{7}$, Josephson junction arrays ${ }^{21}$, colloids or Lennard-Jones systems ${ }^{22}$. However, the disorder mechanism-the way in which disorder proliferates at zero temperature-has not been observed directly. Disorder-induced order has been recently proposed when quenched disorder breaks the continuous 2D symmetry, for example, by introducing a 1D periodic disorder potential ${ }^{3}$. Within this scenario, true long-range order may be favoured by the $1 \mathrm{D}$ disorder, breaking the MWH theorem. Calculations show the stabilization of the quantum Hall ferromagnetic state in graphene monolayers due to strain-induced easy-plane anisotropy ${ }^{23}$ or improved control of the relative phase in randomly coupled condensates $^{24}$. The experimental realization of such a disorderinduced order in the absence of thermal fluctuations has not yet been reported. The effect of symmetry breaking on microscopic properties and the critical exponents of the order-disorder transition are unknown.

Here we address these questions by directly imaging the order-disorder transition in a $2 \mathrm{D}$ vortex lattice induced by a $1 \mathrm{D}$ periodic potential using scanning tunnelling microscopy at $0.1 \mathrm{~K}$. By changing the magnetic field, we modify the coupling strength between the $1 \mathrm{D}$ periodic potential_-produced by a surface corrugation with period $w$-and the vortex lattice, as well as the intervortex distance $a_{0}=(3 / 4)^{1 / 4}\left(\phi_{0} / B\right)^{1 / 2}$ (see Methods and Supplementary Information for a detailed description of sample preparation and the experimental procedure). This allows us to go from a locked $2 \mathrm{D}$ solid, where the lattice is commensurate

\footnotetext{
${ }^{1}$ Laboratorio de Bajas Temperaturas, Departamento de Física de la Materia Condensada, Instituto de Ciencia de Materiales Nicolás Cabrera, Condensed Matter Physics Center (IFIMAC), Universidad Autónoma de Madrid, E-28049 Madrid, Spain, 2 Unidad Asociada de Bajas Temperaturas y Altos Campos Magnéticos, UAM, CSIC, Cantoblanco, E-28049 Madrid, Spain, ${ }^{3}$ H.H. Wills Physics Laboratory, University of Bristol, Tyndall Avenue, Bristol BS8 1TL, UK, ${ }^{4}$ Laboratorio de Microscopías Avanzadas (LMA), Instituto de Nanociencia de Aragón (INA), Universidad de Zaragoza, E-50018 Zaragoza, Spain, ${ }^{5}$ Departamento de Física de la Materia Condensada, Universidad de Zaragoza, E-50009 Zaragoza, Spain, ${ }^{6}$ Instituto de Ciencia de Materiales de Aragón (ICMA), Universidad de Zaragoza-CSIC, Facultad de Ciencias, Zaragoza E-50009, Spain. †Present address: Department of Applied Physics, Eindhoven University of Technology, PO Box 513, 5600 MB Eindhoven, The Netherlands. *e-mail: isabel.guillamon@uam.es
} 


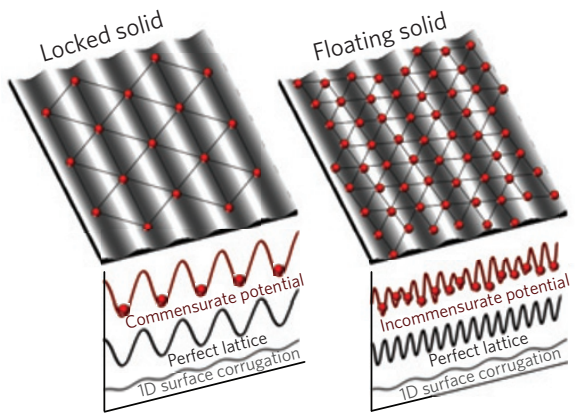

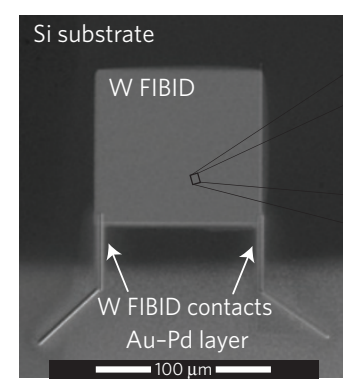

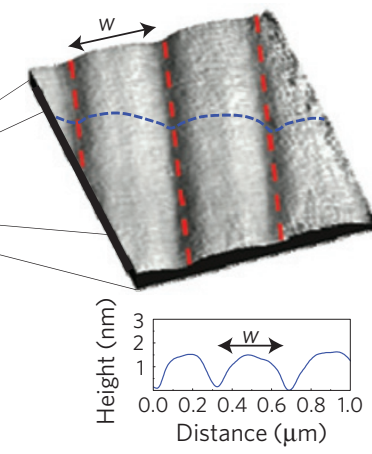

c

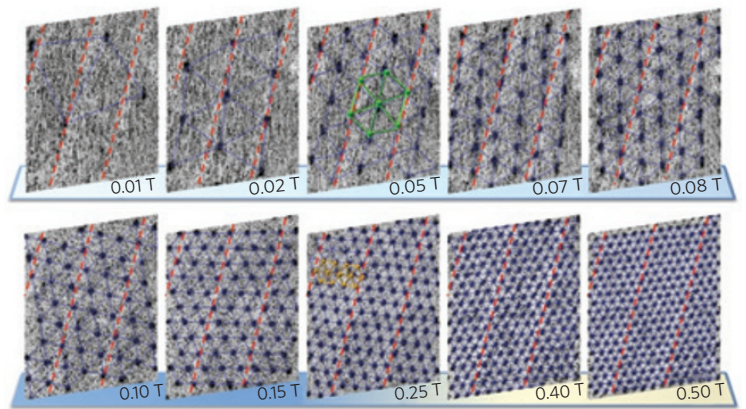

d

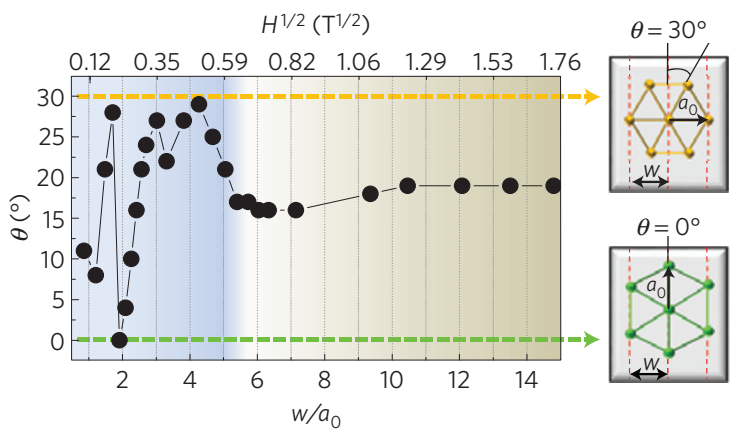

Figure 1 | Unlocking of the 2D vortex lattice on a linear potential. a, Sketch of a commensurate arrangement locked to the 1D potential for small $p$ (left) and an unlocked lattice producing a floating solid incommensurate with the 1D modulation for large $p$ (right). b. Scanning electron microscopy image of the sample (left). STM topography of a $1 \times 1.2 \mu \mathrm{m}^{2}$ area (right) with the profile along the blue dashed line given below. Red dashed lines indicate the 1D modulation. c, Sequence of vortex lattice images taken at $0.1 \mathrm{~K}$ with increasing magnetic field up to $0.5 \mathrm{~T}$ (see Supplementary Movie 1 for the entire sequence). Red dashed lines indicate the 1D modulation and vortices are shown as black points. Blue lines are Delaunay triangulation. d, Dependence on magnetic field of the angle between the 1D modulation and the vortex lattice. Lines joining points are guides to the eye. When $p<5(H<0.4 \mathrm{~T} ;$ blue background), the vortex lattice oscillates between the two primary commensurate arrangements $\theta=0^{\circ}$ (green dotted line) and $\theta=30^{\circ}$ (yellow dotted line). This satisfies, respectively, $w=n \sqrt{3} a_{0} / 2$ and $w=m a_{0}$, with $n$ and $m$ integers (the $n=1$ and $m=1$ cases are highlighted in the upper (yellow) and lower (green) right panels of $\mathbf{d}$, whereas the $n=1$ and $m=4$ commensurate arrangements are identified in the vortex images of $\mathbf{c}$ at respectively $0.05 T$ and $0.25 \mathrm{~T}$ ). At fields above $0.4 \mathrm{~T}(w>p$; khaki coloured in $\mathbf{c}$ and $\mathbf{d})$, the lattice unlocks and adopts an orientation independent of the 1D potential.

with the 1D potential (Fig. 1a, left), to a floating $2 \mathrm{D}$ solid at larger densities, where it becomes incommensurate with the $1 \mathrm{D}$ modulation (Fig. 1a, right).

The coupling strength between the vortex lattice and the 1D modulation depends on the commensurability ratio $p$, defined as $p=w / a_{0}$, and $\theta$ the relative orientation between them (ref. 25). Figure 1c shows a sequence of vortex lattice images obtained at lower magnetic fields. Below $0.4 \mathrm{~T}$, with $p \lesssim 5$, the lattice suffers a series of commensurate to incommensurate transitions that produces a rotation of its overall orientation between $\theta=0^{\circ}$ and $30^{\circ}$, while maintaining a perfect hexagonal arrangement ${ }^{26}$. Figure $1 \mathrm{~d}$ shows $\theta$ as a function of $p$ for vortex lattice images taken with increasing magnetic field. On increasing $p$ above $0.4 \mathrm{~T}(p \gtrsim 5)$, the rotation of the vortex lattice ceases and its orientation becomes independent of the $1 \mathrm{D}$ potential. The lattice is incommensurate with the $1 \mathrm{D}$ potential and forms a floating $2 \mathrm{D}$ solid.

In Fig. 2a we show a sequence of vortex lattice images between $0.5 \mathrm{~T}$ and $5.5 \mathrm{~T}$. We identify three different phases. In phase I, between $0.5 \mathrm{~T}$ and $2 \mathrm{~T}$, there are no topological defects, and all vortices are surrounded by six nearest neighbours. However, the vortex positions show small deviations from those expected for a perfect hexagonal lattice, which become gradually more pronounced on increasing the magnetic field. Between $2.5 \mathrm{~T}$ and $4.5 \mathrm{~T}$, in phase II, we observe the appearance of dislocations, that is pairs of five-fold and seven-fold coordinated vortices. We identify bound dislocation pairs as well as isolated dislocations. Above $4.5 \mathrm{~T}$, in phase III, the density of dislocations increases strongly and we identify the appearance of free disclinations in the form of isolated five-fold or seven-fold coordinated vortices. The images at $5 \mathrm{~T}$ and 5.5 $\mathrm{T}$ show that defects exist over the whole sample, producing a disordered vortex lattice.

One important observation-the appearance of fluctuations in the local vortex density $\rho$-is shown in Fig. 2 b,c. The standard deviation in $\rho$ increases with the field-induced proliferation of defects-from less than $5 \%$ in phase I to up to $20 \%$ at $5.5 \mathrm{~T}$ in phase III (Fig. 2c). Density fluctuations are characteristic of a long wavelength or fully uncorrelated quenched disorder potential ${ }^{27}$.

To further quantify the spatial dependence of vortex disorder, we calculate the translational and orientational correlation functions, $G_{\mathrm{K}}(r)$ and $G_{6}(r)$ (Methods and Supplementary Information). Figure 3a shows the evolution of $G_{\mathrm{K}}(r)$ and $G_{6}(r)$ with the magnetic field. In phase I, between $0.5 \mathrm{~T}$ and $2 \mathrm{~T}$, we observe that $G_{6}(r)$ remains close to 1 and is independent of the distance, whereas $G_{\mathrm{K}}(r)$ decays following a power-law dependence, $G_{\mathrm{K}}(r) \sim r^{-\eta}$, with $\eta_{\mathrm{K}}$ increasing with field. Above $2 \mathrm{~T}$, in phase II, $G_{\mathrm{K}}(r)$ decays exponentially at large distances when $\eta_{\mathrm{K}}=1 / 3$, when a finite amount of defects starts to be observed in the images. Here, $G_{6}(r)$ shows a power-law decay, $G_{6}(r) \sim r^{-\eta_{6}}$, with $\eta_{6}$ increasing continuously from $2 \mathrm{~T}$ up to $4.5 \mathrm{~T}$. In phase III, above $5 \mathrm{~T}, G_{6}(r)$ decays exponentially when $\eta_{6}=1 / 4$, and the defect density diverges-reaching 0.4 -indicating that nearly half of all vortices have five or seven nearest neighbours at $5.5 \mathrm{~T}$ (Fig. 3b). The observed behaviour follows the microscopic twostep sequence for the proliferation of disorder described by BKTHNY theory, with critical values for the exponents $\eta_{\mathrm{K}}^{c}=1 / 3$ and $\eta_{6}^{c}=1 / 4($ ref. 28). 


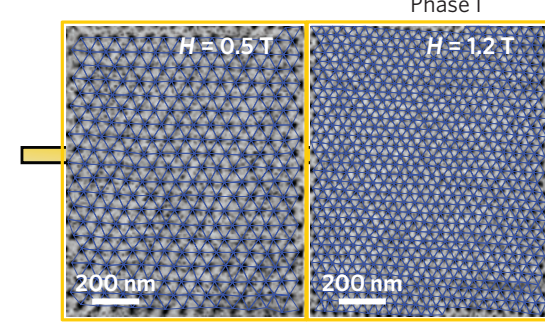

Phase II

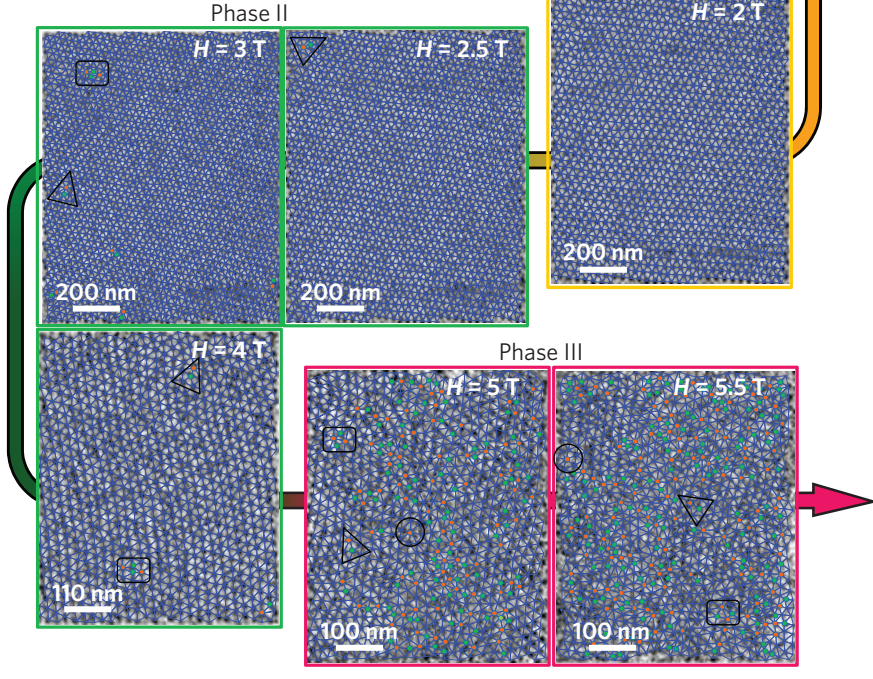

b

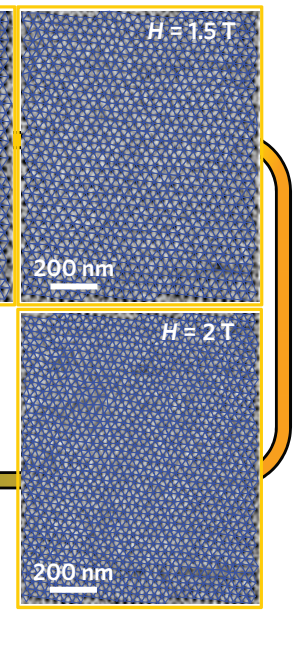

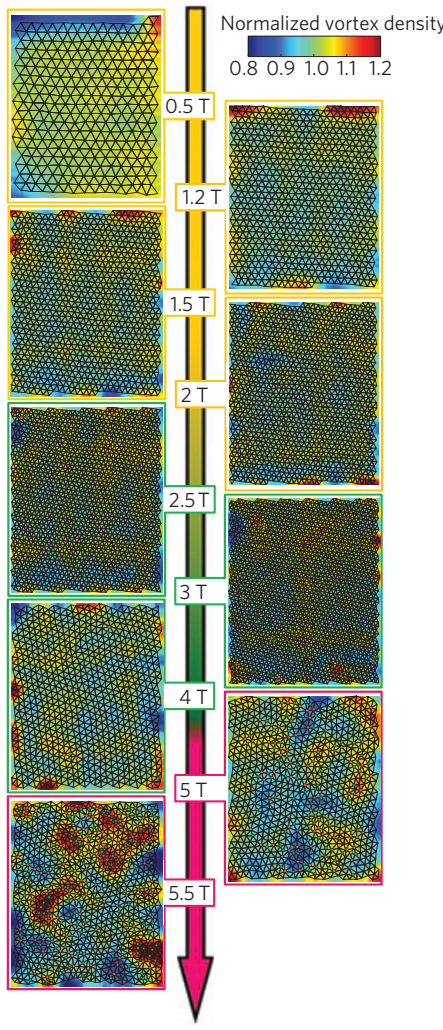

C

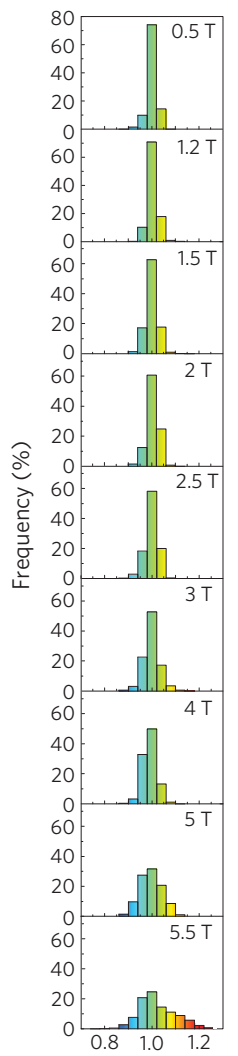

Normalized vortex density

Figure 2 | Order-disorder transition in the 2D vortex lattice at $0.1 \mathrm{~K}$. a, Vortex lattice images taken at $0.1 \mathrm{~K}$ on increasing the magnetic field from $0.5 \mathrm{~T}$ to 5.5 T (see Supplementary Movie 2). Vortices are shown by black points and the Delaunay triangulated lattice as blue lines. Vortices with five and seven nearest neighbours are identified by green and orange points. Dislocations formed by five and seven nearest neighbour pairs of vortices are identified by black triangles, pairs of dislocations by black rectangles and isolated disclinations by black circles. Above $4 \mathrm{~T}$, the image size is decreased to better resolve individual vortices. $\mathbf{b}$, Positional fluctuations of the vortex density during the disorder process calculated from the vortex images shown in $\mathbf{a}$. Colour scale is given at the top right (see Section III.3 in Supplementary Information). Deviations in the local vortex density become stronger with increasing magnetic field. $\mathbf{c}$, Histograms of the vortex density obtained from the images in $\mathbf{b}$ using the same colour scale.

To investigate the microscopic disorder mechanism, we further analyse the first entrance of disorder in the ordered phase I. Deviations in the vortex positions with respect to a perfect hexagonal lattice can be quantified by the relative displacement correlator $B(r)$ (given by $B(r)=\left\langle[\mathbf{u}(\mathbf{r})-\mathbf{u}(0)]^{2}\right\rangle / 2$, where $\mathbf{u}(\mathbf{r})=\mathbf{r}-\mathbf{r}_{p}$ is the displacement of each vortex at $\mathbf{r}$ from its position in the perfect lattice $\mathbf{r}_{p}$; see ref. 27). We find that $B(r)$ grows as $\ln \left(r / a_{0}\right)$ in the dislocation-free Phase I. In Fig. 3c we plot the result at $1.5 \mathrm{~T}$. In $2 \mathrm{D}$ systems, this is the expected behaviour in response to a scale-invariant disorder ${ }^{19}$. Next, we fit the data using the Gaussian approximation $G_{\mathrm{K}}(r)=\mathrm{e}^{-K^{2} B(r) / 2}$ (valid for Gaussian disorder potentials) and the translational correlation function $G_{\mathrm{K}}(r)$ (shown in Fig. 3a). A comparison reveals a very good agreement (Fig. 3c). Therefore, all three independent observations (local vortex density fluctuations, logarithmic growth of $B(r)$, and a Gaussian distribution of displacements) show that a random potential drives the transition.

We next focus on the source of scale-invariant disorder driving the transition. No thermally induced or quantum-induced fluctuations are available to effectively disorder the vortex lattice here. At $0.1 \mathrm{~K}$, the transition induced by either thermal or quantum fluctuations is expected to occur at a magnetic field extremely close to $H_{\mathrm{c} 2}$ (see Supplementary Information). Our sample is amorphous and compositionally homogeneous, both laterally and across its thickness, thus, there is no quenched disorder from compositional or structural changes. Instead, thickness variations given by the $1 \mathrm{D}$ modulation emerge as the only source for quenched disorder. The fundamental property of a scale-invariant potential $V(r)$ is that it has long-range logarithmic correlations ${ }^{6}$

$$
\left\langle\left[V(r)-V\left(r^{\prime}\right)\right]^{2}\right\rangle=4 \sigma J^{2} \ln \left(r-r^{\prime}\right)
$$

where $J=\mu d a_{0}^{2} / 2 \pi$ is the elastic interaction strength (the magnetic field dependence of the shear modulus $\mu$ is discussed in the Supplementary Information) and $\sigma$ is the disorder strength. In Fig. 3d we calculate the spatial correlations of $V(r)$ (the first term in equation (1)) by taking $V_{1 \mathrm{D}}(r)=z(r) \varepsilon_{L}$, where $z(r)$ is the topography and $\varepsilon_{L}=\left(\phi_{0}^{2} / 4 \pi \mu_{0} \lambda^{2}\right) \ln (\lambda / \xi)$ is the vortex energy per unit length (see Supplementary Information for details). We find that $V_{1 \mathrm{D}}(r)$ has long-range logarithmic correlations and shortrange smooth periodic correlations at integer multiples of $w$, which are strongly damped at large distances. Thus, incommensurate 1D correlation behaves as a quasi-random disorder potential.

We can write the free energy, $F$, following available renormalization group $(\mathrm{RG})$ theory for random disorder $a s^{6,7}$ :

$$
F=-2 T \ln (L)+J \ln (L)-J \sqrt{\sigma / \sigma_{\mathrm{c}}} \ln (L)
$$

where the thermal energy $E_{\mathrm{th}}$, elastic energy $E_{\mathrm{el}}$, and disorder energy $E_{\text {dis }}$ (first to third terms, respectively) diverge logarithmically with the system size $L$ (ref. 6,7). Here, $\sigma_{\mathrm{c}}$ is the critical value for the 


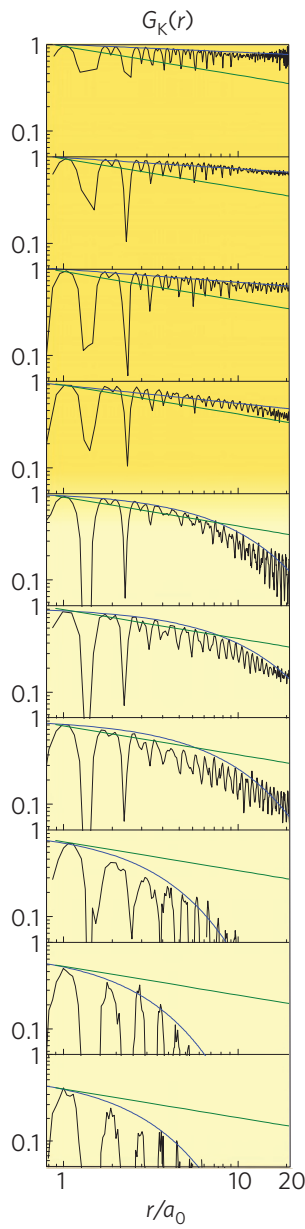

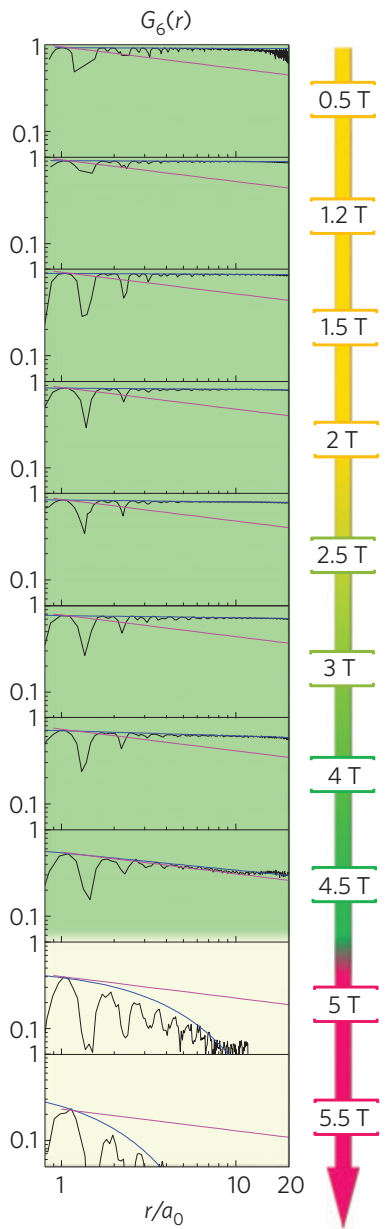

b

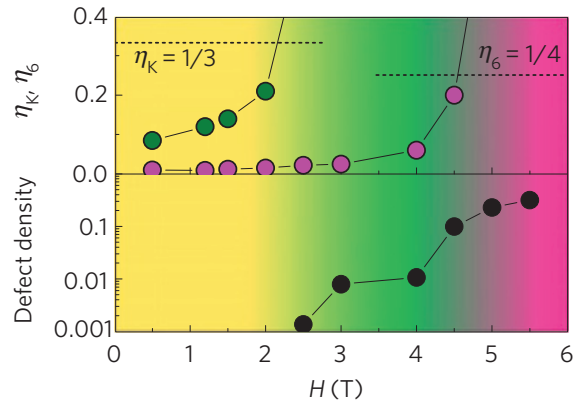

C

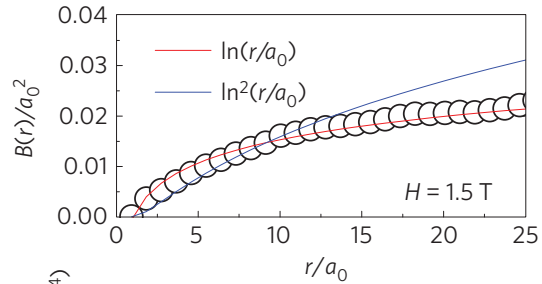

d

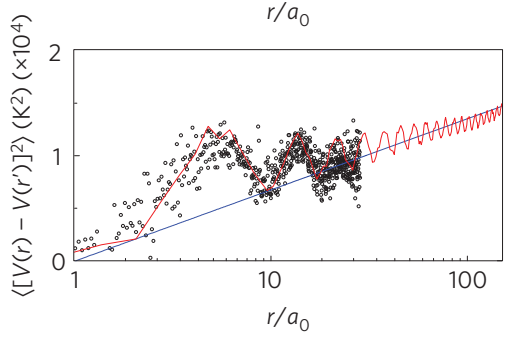

e

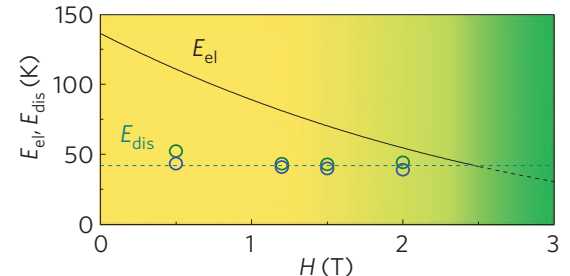

Figure 3 | Correlation functions and critical exponents. a, Field-induced changes in the correlation functions for translational order $G_{K}(r)$ (left) and orientational order $G_{6}(r)$ (right) between $0.5 \mathrm{~T}$ and $5.5 \mathrm{~T}$. Blue lines in the graphs are the fits for the envelopes of the correlation functions (black lines). Green (left) and magenta (right) lines stand for power decay laws with, respectively, the critical exponents $\eta_{\mathrm{K}}^{\mathrm{c}}=1 / 3$ and $\eta_{6}^{\mathrm{c}}=1 / 4$. The background colour (yellow for $G_{K}(r)$ and green for $G_{6}(r)$ ) represents the range of the order and turns from dark (long-range) to light (short-range) when the exponents $\eta_{K}$ and $\eta_{6}$ become higher than the critical values and the correlation functions change from a power law to exponential decay with the distance $r$. $\mathbf{b}$, (Top) Field dependence of the power-law decay exponents $\eta_{K}$ (green points) and $\eta_{6}$ (magenta points). (Bottom) Density of five-fold and seven-fold coordinated vortices as a function of the magnetic field. c, Correlation function $B(r) / a_{0}^{2}$ at $1.5 \mathrm{~T}$ (black dots, see text and Supplementary Information for details). Red line is $\approx\left(3 \eta_{K} / 8 \pi^{2}\right) \ln \left(r / a_{0}\right)$, obtained within the Gaussian approximation for a scale-invariant random potential (see text and Supplementary Section $\mathrm{VI}$ ). Blue line gives the $\ln ^{2}(r)$ dependence expected within correlated pinning disorder. d, Spatial dependence of the mean-squared correlation of the 1D surface potential $\left\langle\left[V_{1 D}(r)-V_{1 D}\left(r^{\prime}\right)\right]^{2}\right\rangle$ at 1.2T, where $V_{1 D}(r)$ is as defined in the text and $r, r^{\prime}$ are the actual vortex positions obtained from the vortex image at $1.2 T$ (see Section III.5 in Supplementary Information for details of the calculations). The red line was obtained by simulating the topography with a periodic modulation and extrapolating the vortex position in an area 100 times larger than in the experiment (see Supplementary Information). The blue line is the fit to equation (1) in the main text. e, Magnetic field dependence of $E_{\mathrm{el}}$ (black line) and $E_{\text {dis }}$ (blue and green circles). $E_{\text {dis }}$ (blue dashed line) is estimated independently from power-law exponents $\eta_{\mathrm{K}}$ in positional correlation (green circles) and long-range logarithmic correlations of $V_{1 \mathrm{D}}(r)$ (blue circles).

disorder strength. In the ordered phase at low temperatures, the relative strength between $E_{\mathrm{el}}$ and $E_{\mathrm{dis}}$ determines the algebraical decay of the translational correlations, with the exponent $\eta_{\mathrm{K}}$ in a hexagonal solid given by ${ }^{19,21}$,

$$
\eta_{\mathrm{K}}=\frac{2}{3}\left[\frac{T}{J}+\sigma\right]
$$

Following the transition from power law to exponential decay in $G_{\mathrm{K}}(r)$, we found $\eta_{\mathrm{K}}^{\mathrm{c}}=1 / 3$ (Fig. 3a), which corresponds to $\sigma_{\mathrm{c}}=1 / 2$. We now calculate the value of $E_{\text {dis }}$ produced by $V_{\mathrm{lD}}(r)$ (using equations (1) and (2)) and, independently, from the power-law decay of the positional correlation functions (using equations (2) and (3) with the $\eta_{\mathrm{K}}$ values from Fig. 3b). The results obtained by the two methods are plotted in Fig. 3e (as blue and green circles, respectively) together with $E_{\text {el }}$ (black line) versus the magnetic field. Note that $E_{\text {th }}$ at $0.1 \mathrm{~K}$ is three orders of magnitude smaller than both $E_{\mathrm{el}}$ and $E_{\mathrm{dis}}$, so it is negligible here. The agreement between $E_{\mathrm{dis}}$ determined from the exponents in the correlation functions $\eta_{\mathrm{K}}$ (green circles) and from logarithmic correlations in the 1D disorder potential (blue circles) is almost perfect. This demonstrates that the 1D surface corrugation produces the disorder through incommensuration and provides the energy scale for the random field driving the transition in the $2 \mathrm{D}$ lattice. Figure $3 \mathrm{e}$ shows that $E_{\text {dis }}$ begins to exceed $E_{\mathrm{el}}$ at the magnetic field where we start to observe dislocations in the images.

Finally, let us discuss the critical behaviour of the observed transitions. Our experiments closely reproduce the expected 
features for the zero-temperature phases as induced by random disorder, with, in particular, positional fluctuations which increase as $\ln (r)$ below the critical disorder and correlations that decay exponentially for high disorder strengths. But here we find critical values, $\sigma_{\mathrm{c}}=1 / 2$ and $\eta_{\mathrm{K}}^{\mathrm{c}}=1 / 3$, which are far above those proposed in theory on the basis of RG calculations and models for random quenched disorder $\left(\sigma_{\mathrm{c}}=1 / 8\right.$ and $\eta_{\mathrm{K}}^{\mathrm{c}}=1 / 12$; refs $\left.6,7,18,29\right)$. The difference between random field theories and our experiments is the presence of symmetry-breaking $1 \mathrm{D}$ modulation. A recent proposal shows that the XY model with 1D symmetry-breaking disorder has an increased order parameter at all temperatures ${ }^{3}$. An earlier work also points out that correlations in the disorder potential enhance the critical value of $\sigma$ (ref. 21). This strongly suggests that the $1 \mathrm{D}$ modulation, by breaking symmetry, modifies the screening of the interactions among dislocations to enhance the critical point and exponents with respect to random field theories.

Our experiments show that, in the presence of the 1D symmetrybreaking disorder, the critical exponents increase to the values expected by BKTHNY theory and the microscopic disordering behaviour follows the sequence defined by the two-step thermal melting transition, suggesting that this route for creating disorder possibly describes more phenomena than just $2 \mathrm{D}$ thermal melting. Inherent to this is the presence both of an intermediate hexatic phase and bound dislocations in the ordered phase which are not expected within random field model ${ }^{29,30}$. The question is: why does our experiment follow BKTHNY theory? To answer this question, one needs to calculate new critical points of the order-disorder transition at zero temperature by taking into account symmetry-breaking correlations within randomness and their influence on the renormalization of the parameters involved in the transition.

Overall, our data represent the first evidence that incommensurate $1 \mathrm{D}$ modulation widens the stability range of the ordered phase in $2 \mathrm{D}$ systems at zero temperature and raise questions that will motivate a detailed examination of the effect of correlations in the critical behaviour of disordered systems. 2D random environments are usually unavoidable in different fields, such as colloids, optical lattices, quantum condensates, $2 \mathrm{D}$ crystals or graphene. The experimental approach presented here reveals an exciting new opportunity to produce coherence in the presence of $1 \mathrm{D}$ symmetry-breaking fields, as for example nematicity.

\section{Methods}

Sample. Our sample is an ultraflat amorphous thin film with a thickness, $d$, of $200 \mathrm{~nm}$ and a lateral size of $100 \mu \mathrm{m}$, fabricated by focused-ion-beam-induced deposition (microscopy and nanofabrication methods are described in detail in sections I and II of the Supplementary Information). $d$ is far below $L_{c}$, the characteristic length for vortex bending along the field direction; thus the vortex lattice forms a $2 \mathrm{D}$ solid. The surface roughness is less than $1 \%$ of $d$ and consists of a smooth 1D modulation with period $w=400 \mathrm{~nm}$ (Fig. 1b).

Low-temperature STM/S vortex imaging. We use scanning tunnelling microscopy/spectroscopy (STM/S) to directly determine modifications in the spatial correlations induced by disorder in the vortex lattice and visualize the microscopic details of the ordered and disordered phases. The STM is carried out in a dilution refrigerator and we work at temperatures low enough $(0.1 \mathrm{~K})$ to neglect any temperature-induced effects. The sample is biased through W-based contact pads, as shown in Fig. 1b (see Supplementary Information). The order-disorder transition was followed by imaging up to thousands of individual vortices from fields of $0.01 \mathrm{~T}$ up to just below $H_{\mathrm{c} 2}$, with the vortex density increasing by a factor of 500 . The average intervortex distance $a_{0}$ decreases with field, following the expected dependence in a triangular vortex lattice (shown in Section III.3 of Supplementary Information).

Locked and floating vortex lattice. We modify the interaction strength between the vortex lattice and the underlying disorder potential by increasing the magnetic field (see Section V in Supplementary Information). At low magnetic fields, when the lattice constant and disorder wavelength are similar (small $p$ ), commensurate vortex configurations are observed, as expected, at matching conditions $p=n$ or $n \sqrt{3} / 2$, with $n$ an integer, and $\theta=30^{\circ}$ or $0^{\circ}$ degrees, respectively ${ }^{26}$. Generally, commensurate lattices locked to the 1D potential are favoured, because they lower the elastic energy of the lattice (Fig. 1). At higher fields, when the lattice constant is much smaller than the 1D modulation, the increase in elastic energy obtained by adjusting to the potential decreases and the lattice can show incommensurate configurations forming a floating solid (Figs 1 and 2).

Calculation of the correlation functions of the vortex images. The order-disorder transition has been characterized in real space through the calculation of translational and orientational correlation functions, $G_{\mathrm{K}}(r)$ and $G_{6}(r)$, vortex density fluctuations $\rho(r)$, and the relative displacement correlator $B(r)$ (see details for the calculation in Sections III.2, III.3 and VI of Supplementary Information). $G_{\mathrm{K}}(r)$ and $G_{6}(r)$ are directly obtained from the individual vortex positions in the images. Peaks in the correlation functions appear at the distances to $n$th nearest neighbours. Deviations with respect to the perfect lattice produce a decay with $r$ of the envelopes of $G_{\mathrm{K}}(r)$ and $G_{6}(r)$ that describes, respectively, weakening of translational and orientational correlations. The crossovers between phases I (yellow), II (green) and III (magenta) are determined as the fields at which the translational and orientational order become short range and the exponents $\eta_{\mathrm{K}}$ and $\eta_{6}$ reach the critical values (dotted lines in Fig. 3b, see text for further details). The relative displacement correlator $B(r)$ is calculated by minimizing the averaged relative deviation between the experimental vortex positions and the simulated perfect hexagonal lattice (see Section VI in Supplementary Information for details of the calculations and $B(r)$ at different fields in Phase I). The evolution of the disordering process in reciprocal space is shown in Section IV of the Supplementary Information through the gradual changes of the height and width of the vortex lattice Bragg peaks.

Received 10 January 2014; accepted 17 September 2014; published online 26 October 2014

\section{References}

1. Anderson, P. W. Absence of diffusion in certain random lattices. Phys. Rev. 109, 1492-1505 (1958).

2. Fisher, M., Weichman, P., Grinstein, G. \& Fisher, D. Boson localization and the superfluid-insulator transition. Phys. Rev. B 40, 546-570 (1989).

3. Wehr, J., Niederberger, A., Sanchez-Palencia, L. \& Lewenstein, M. Disorder versus the Mermin-Wagner-Hohenberg effect: From classical spin systems to ultracold atomic gases. Phys. Rev. B 74, 224448 (2006).

4. Billy, J. et al. Direct observation of Anderson localization of matter waves in a controlled disorder. Nature 453, 891-894 (2008).

5. Sanchez-Palencia, L. \& Lewenstein, M. Disordered quantum gases under control. Nature Phys. 6, 87-95 (2010).

6. Nattermann, T., Scheidl, S., Korshunov, S. \& Li, M. Absence of reentrance in the two-dimensional XY-model with random phase shift. J. Phys. 5, 565-572 (1995)

7. Cha, M-C. \& Fertig, H. A. Disorder-induced phase transition in two-dimensional crystals. Phys. Rev. Lett. 74, 4867-4870 (1995).

8. Nelson, D. R. \& Halperin, B. I. Dislocation-mediated melting in two dimensions. Phys. Rev. B 19, 2457-2484 (1979).

9. Berezinskii, V. Destruction of long-range order in one-dimensional and two-dimensional systems possessing a continuous symmetry group. II. Quantum Systems. Sov. Phys. JETP 34, 610-616 (1972).

10. Kosterlitz, J. M. \& Thouless, D. J. Ordering, metastability and phase transitions in two-dimensional systems. J. Phys. C 6, 1181-1203 (1973).

11. Carpentier, D. \& Doussal, P. L. Melting of two-dimensional solids on disordered substrates. Phys. Rev. Lett. 81, 1881-1884 (1998).

12. Nattermann, T. Scaling approach to pinning: Charge density waves and giant flux creep in superconductors. Phys. Rev. Lett. 64, 2454-2457 (1990).

13. Minnhagen, P. The two-dimensional Coulomb gas, vortex unbinding, and superfluid-superconducting films. Rev. Mod. Phys. 59, 1001-1066 (1987).

14. Mermin, N. D. Crystalline order in two dimensions. Phys. Rev. 176, 250-254 (1968)

15. Hohenberg, P. Existence of long-range order in one and two dimensions. Phys. Rev. 158, 383-366 (1967).

16. Halperin, B. I. \& Nelson, D. R. Theory of two-dimensional melting. Phys. Rev. Lett. 41, 121-124 (1978).

17. Young, A. P. Melting and the vector Coulomb gas in two dimensions. Phys. Rev. B 19, 1855-1866 (1979).

18. Nelson, D. R. Reentrant melting in solid films with quenched random impurities. Phys. Rev. B 27, 2902-2914 (1983).

19. LeDoussal, P. \& Giamarchi, T. Dislocation and Bragg glasses in two dimensions. Physica C C331, 233-240 (2000).

20. Guillamon, I. et al. Direct observation of stress accumulation and relaxation in small bundles of superconducting vortices in tungsten thin films. Phys. Rev. Lett. 106, 077001 (2011). 
21. Korshunov, S. \& Nattermann, T. Phase diagram of a Josephson junction array with positional disorder. Physica B 222, 280-286 (1996).

22. SadrLahijany, M., Ray, P. \& Stanley, H. Dispersity-driven melting transition in two-dimensional solids. Phys. Rev. Lett. 79, 3206-3209 (1997).

23. Abanin, D., Lee, P. \& Levitov, L. Randomness-induced XY ordering in a graphene quantum Hall ferromagnet. Phys. Rev. Lett. 98, 156801 (2007).

24. Niederberger, A. et al. Disorder-induced order in two-component Bose-Einstein condensates. Phys. Rev. Lett. 100, 030403 (2008).

25. Radzihovsky, L., Frey, E. \& Nelson, D. Novel phases and reentrant melting of two-dimensional colloidal crystals. Phys. Rev. E 63, 031503 (2001).

26. Martinoli, P. Static and dynamic interaction of superconducting vortices with a periodic pinning potential. Phys. Rev. B 17, 1175-1194 (1978).

27. Giamarchi, T. \& LeDoussal, P. Elastic theory of flux lattices in the presence of weak disorder. Phys. Rev. B 52, 1242-1270 (1995).

28. Zahn, K., Lenke, R. \& Maret, G. Two-stage melting of paramagnetic colloidal crystals in two dimensions. Phys. Rev. Lett. 82, 2721-2724 (1999).

29. Carpentier, D. \& Doussal, P. L. Topological transitions and freezing in XY models and Coulomb gases with quenched disorder: Renormalization via traveling waves. Nucl. Phys. B 588, 565-629 (2000).

30. Herrera-Velarde, S. \& von Grünberg, H. H. Disorder-induced vs temperature-induced melting of two-dimensional colloidal crystals. Soft Matter 5, 391-399 (2009).

\section{Acknowledgements}

This work was supported by the Spanish MINECO (FIS2011-23488,

MAT2011-27553-C02, MAT 2012-38318-C03, Consolider Ingenio Molecular

Nanoscience CSD2007-00010), the Comunidad de Madrid through program

Nanobiomagnet (S2009/MAT-1726) and by the Marie Curie Actions under the project

FP7-PEOPLE-2013-CIG-618321 and contract no. FP7-PEOPLE-2010-IEF-273105. We acknowledge the technical support of UAM's workshop SEGAINVEX.

\section{Author contributions}

I.G. carried out the experiment, analysis and interpretation of data. I.G. wrote the paper together with H.S. and S.V. Samples were made and characterized by R.C. and J.S.

J.M.D.T. and M.R.I. supervised the sample design and fabrication. All authors discussed the manuscript text and contributed to it.

\section{Additional information}

Supplementary information is available in the online version of the paper. Reprints and permissions information is available online at www.nature.com/reprints.

Correspondence and requests for materials should be addressed to I.G.

\section{Competing financial interests}

The authors declare no competing financial interests. 